\title{
Model and Experiment on Resistance Loss of Wet Chordal Grid with Spray Pressure and Structural Parameters
}

\author{
Hua Guo $\mathbb{D D}^{1,2}$ Haiqiao Wang, ${ }^{1}$ and Zhirong $W u^{1}$ \\ ${ }^{1}$ School of Resource, Environment and Safety Engineering, Hunan University of Science and Technology, Xiangtan 411201, \\ Hunan, China \\ ${ }^{2}$ School of Civil and Environmental Engineering, Hunan University of Science and Engineering, Yongzhou 425199, Hunan, China \\ Correspondence should be addressed to Hua Guo; ghaq1989@163.com
}

Received 24 November 2020; Revised 19 December 2020; Accepted 25 January 2021; Published 5 February 2021

Academic Editor: Chiara Bedon

Copyright (C) 2021 Hua Guo et al. This is an open access article distributed under the Creative Commons Attribution License, which permits unrestricted use, distribution, and reproduction in any medium, provided the original work is properly cited.

To reduce the dedusting resistance of wet vibrating string grille precipitators during dedusting in mine ventilation roadways, we investigated the dedusting resistance characteristics of water fog and water film coupling and determined the relationship between dedusting resistance and spray pressure, vibrating grid filling rate, and wind speed. A mathematical resistance coefficient model is established using hydrodynamics theory and capillary mechanics. The theoretical relationship of dedusting resistance is deduced. The results show that when wind speed is constant, the spray pressure and dedusting resistance are higher and the resistance is smaller with a high filling rate compared with a low filling rate. Constant spray pressure allows faster wind speeds and reverse pressure gradient forces to increase when dust flows around the wet vibrating wire, which makes the pressure distribution asymmetrical around the steel wire and increases resistance. Dust removal resistance of the resonance chord with a high filling rate is substantially lower than that with a low filling rate under the same working conditions. On the basis of satisfying the dedusting efficiency, the resonance chord dedusting system does not affect normal production and resistance is low. The spray pressure is controlled at $0.3-0.7 \mathrm{MPa}$ and the optimal wind speed is $3-4 \mathrm{~m} / \mathrm{s}$. According to the theoretical calculation and experimental data, the optimal filling rate of a vibrating string grid plate is $77.8 \%$, spray pressure is $0.7 \mathrm{MPa}$, and wind speed is $3.5 \mathrm{~m} / \mathrm{s}$. Dust removal with low resistance and improved economic benefit can thus be obtained.

\section{Introduction}

Dust pollution is one of the six major disasters in the mining engineering industry [1] and affects the physical and mental health of mine workers. Respirable mining dust does not settle easily, which causes the mine exhaust system to enter the atmosphere and harm the health and living environment of communities surrounding the mining area. With new implementation of an industrial pollutant discharge standard in China [2], the manner by which mine dust is controlled from the source is particularly important [3-5], especially for dust from mine return roadways. Dedusting measures for mine dust have been rapidly developed to address this problem. The main dedusting measures are coal seam water injection, ventilation dedusting, spray dedusting, and dust collector dedusting [6-9]. However, dust removal efficiency remains low, especially for respirable dust [10]. Although wet dedusting equipment can be used to improve dust removal efficiency, implementation is impractical because it affects normal mine production. Exploration of a wet vibrating wire grid dedusting technology with high dedusting efficiency and low resistance is therefore required to resolve the dedusting resistance characteristics of a vibrating string grid in theory and practical engineering experiments.

Coupled dedusting of water mist and water films is a new approach to gas purification. Compared with the conventional wet-fiber grid dedusting method, coupled dedusting has the advantages of high dedusting efficiency (especially for respirable dust), low resistance, low energy consumption, convenient ash cleaning, and simple maintenance. Previous studies on the dedusting resistance of a wet vibrating grid 
have mainly been based on experiments. Gong et al. [11] studied dedusting resistance from the spray state on the grid combined with the number of vibrating chord grid layers and wind speeds. Ge [12] tested three different specifications of fiber grids and water pressures and added dust reducer to the water to investigate dedusting resistance. Zhao [13] analyzed the resistance characteristics of a vibrating chord gate from two aspects of fog droplets and the vibrating string steel wire and experimentally measured the resistance corresponding to different spray quantities. Zhang [14] carried out single-factor experiments on dust removal resistance focusing on spray water quantity, spray distance, grid plate number, and filtration wind speed. Chen and Zhang [15] constructed a dust purification model of a wet-fiber grid and deduced a semiempirical and semitheoretical formula of dust removal resistance suitable for low wind speeds $(1-1.5 \mathrm{~m} / \mathrm{s})$. In addition, wet-fiber grid dedusting has been applied in some mining enterprises and smelters, but the field application resistance is large, and the effect is not ideal. However, there is little theoretical research on resistance characteristics using steel wire based on the coupling action of water mist and the vibrating chord grid and the formation/breakage of the chord gate water film.

We therefore analyze coupled dedusting resistance characteristics of water mist and water film from the basic theories of capillary mechanics, hydrostatics, vibration dynamics, hydrodynamics, and aerosol mechanics and develop a resistance theory model. The friction and inertia resistance of air flowing around the vibrating string steel wire are deduced on the basis of flow theory. A comparison of theoretical calculations and experimental data provides the theoretical basis and application guidance for dust prevention and the control of mine return-air roadways by wet vibrating string grid dedusting technology.

\section{Materials and Methods}

2.1. Friction Resistance and Inertia Resistance. Vibrating gate dedusting under the coupled action of a water mist and water film is the process of gas purification of dust under the force of air flow and water mist through a wet vibrating wire grid plate. The water mist is sprayed uniformly on the grid plate and a water film forms under the double action of capillary and resonance. Flow resistance is the flow through the interface (Figure 1), which causes a change in energy. When a fluid such as water, wind, or aerosol flows around a cylindrical vibrating wire, the asymmetric shear stress on the wire surface and pressure change present important controlling factors of flow resistance. The pressure difference is the inertia resistance, and the shear force is the friction resistance.

\subsection{Establishment and Derivation of Resistance Theory Model}

\subsubsection{Basic Hypothesis}

(1) The cross section of the wire in the vibrating wire grid is circular.
(2) The water mist from the nozzle flows perpendicular along the wire under airflow carrying dust particles.

(3) There is no heat exchange on the surface of the wire, which is an adiabatic wall.

(4) The water mist through the wire is uniformly distributed and the water film that forms in the wire is also uniformly distributed in the entire wire gap of the grid plate.

(5) The dust particles captured on the vibrating string panel are removed with the flow of water mist or rupture of the water film, and the subsequent dedusting process remains unaffected.

2.2.2. Theoretical Model. The microelement of the vibrating wire layer is taken along the flow field streamline $M$ direction, as shown in Figure 2. Force analysis of the microelement body shows that the forces acting on the microplasma along the air flow direction are as follows: the friction resistance $f$ of the fluid flowing through the gap in the vibrating wire and the inertia resistance $\Delta P_{1}$ caused by the asymmetrical pressure distribution around the wire.

The wire arranged around the two-dimensional air flow structure is regarded as a long cylinder perpendicular to the wind flow direction owing to the gap between the steel wires. The flow around the long cylinder group is parallel. Xiang [16] derived the dimensionless friction resistance formula $f^{*}$ using the cell shell model (shear force free condition):

$$
f^{*}=\frac{4 \pi}{-(1 / 2)-(1 / 2) \ln \beta+\left(\beta^{2} / 1+\beta^{2}\right)},
$$

where $\beta$ is the dimensionless filling rate of wet vibrating wire that can be obtained by

$$
\beta=\beta_{s}+\beta_{w}
$$

where $\beta_{s}$ is the dimensionless wire filling rate, that is, the ratio of wire area to chord grid area, $\beta_{w}$ is the water filling rate on the wire, and it can be calculated by measuring the latter's liquid carrying capacity [17].

The dimensionless friction resistance $f^{*}$ can be derived from the principle of classical mechanics. Specifically, the friction resistance of the grid plate to the wetting air flow obeys Newton's law [18] if the friction resistance acting on the wire per unit length is assumed to be

$$
f^{*}=\frac{f}{v \mu},
$$

where $v$ is the air velocity $(\mathrm{m} / \mathrm{s})$ and $\mu$ is the aerodynamic viscosity $(\mathrm{Pa} \cdot \mathrm{S})$.

When fluid flows around the vibrating wire, the aerodynamic viscosity can be expressed as [19]

$$
\mu=\mu_{0}\left(\frac{T}{288.15}\right)^{(3 / 2)} \frac{288.15+B}{T+B},
$$

where $\mu_{0}$ is the dynamic viscosity of air at $15^{\circ} \mathrm{C}$, $1.79 \times 10^{-5} \mathrm{~Pa} \cdot \mathrm{S}, \mathrm{T}$ is the gas temperature $(\mathrm{K}), 110.4 \mathrm{~K}$, and $B$ is a constant related to the gas properties. 


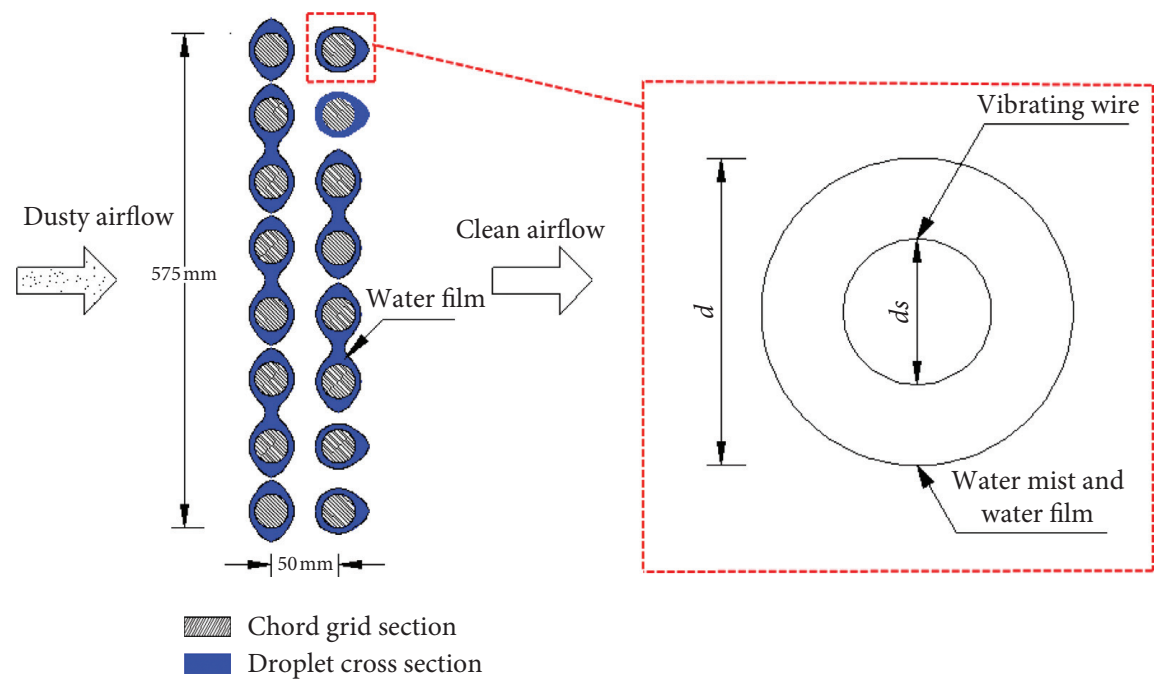

FIGURE 1: Water fog and vibrating string grille water film dedusting mechanism.

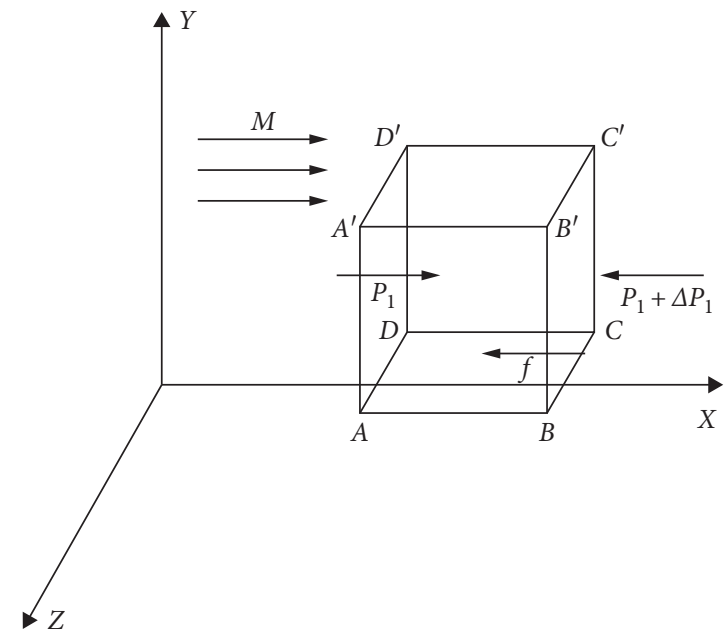

FIGURE 2: Force analysis of a vibrating string grille microelement body.

The flow around a long cylinder has both potential and viscous flows. Viscous flow is mainly considered to calculate $f$, whereas potential flow can be ignored. Equation (3) can be substituted into equation (1). The friction resistance $f$ of fluid flow around a vibrating wire is

$$
f=f^{*} v \mu=\frac{4 \pi v \mu}{-(1 / 2)-(1 / 2) \ln \beta+\left(\beta^{2} / 1+\beta^{2}\right)} .
$$

Combined with equations (4) and (5), the following is obtained:

$$
f=\frac{4 \pi v\left(\left(\left(7.13 \times 10^{-3}\right) /(T+110.4)\right) \times(T / 288.15)^{(3 / 2)}\right)}{-(1 / 2)-(1 / 2) \ln \beta+\left(\beta^{2} / 1+\beta^{2}\right)} .
$$

When moist dust carried by the air flows through the gap of the vibrating wire, it contracts into the gap and the water film in the clearance deforms under viscoelastic action until the water film is blown through. At this point, the cross section at the back of the vibrating wire is minimized, whereas the cross section flow in the rear section is full. The fluid flowing through the wire is approximately regarded as the fluid flowing around the cylinder. The inertia resistance of the fluid flowing through the gap is calculated according to the principle of pressure loss around the flow cylinder. The continuity equation is [20]

$$
\begin{aligned}
& v_{1}=\frac{Q}{A}, \\
& v_{2}=\frac{Q}{\varepsilon A_{0}},
\end{aligned}
$$

where $v_{1}$ is the average velocity of fluid in the vibrating wire gap, $1.79 \times 10^{-5} \mathrm{~m} / \mathrm{s}, Q$ is the flow rate of the wire clearance through the fluid $\left(\mathrm{m}^{3} / \mathrm{s}\right), A$ is the wire clearance area $\left(\mathrm{m}^{2}\right), v_{2}$ 
is the velocity at the minimum shrinkage section between the wire gaps $(\mathrm{m} / \mathrm{s}), \varepsilon$ is the shrinkage coefficient of airflow through the minimum section (dimensionless), and $A_{0}$ is the rupture area when air flows through the water film $\left(\mathrm{m}^{2}\right)$.

The average velocity $v_{1}$ of the vibrating wire gap fluid is expressed as

$$
v_{1}=\frac{v}{1-\beta} .
$$

The Baroda formula [21] is used to study the inertia resistance of the flow through the unit area of the wire gap:

$$
\Delta P_{1}=\frac{1}{2} \rho\left(v_{1}-v_{2}\right)^{2} .
$$

Replacing equation (8) with (9) yields

$$
\Delta P_{1}=\frac{1}{2} \rho\left(\frac{Q}{\varepsilon A_{0}}-\frac{Q}{A}\right)^{2} .
$$

Further deformation includes

$$
\Delta P_{1}=\frac{1}{2} \rho\left(\frac{Q}{\varepsilon A_{0}}-\frac{Q}{A}\right)^{2}\left(\frac{Q}{A}\right)^{2}\left(\frac{A}{Q}\right)^{2},
$$

which can be simplified to

$$
\Delta P_{1}=\frac{1}{2} \rho\left(\frac{A}{\varepsilon A_{0}}-1\right)^{2} v_{1}^{2} .
$$

The following formula is used to obtain $A_{0}$ when air flows through the water film:

$$
A_{0}=A-A_{w}
$$

where $A_{w}$ is the effective water film area (sum of the primary and secondary film areas) $\left(\mathrm{m}^{2}\right)$.

Replacing equation (8) with equations (12) and (13) gives

$$
\Delta P_{1}=\frac{1}{2} \rho\left(\frac{A}{\varepsilon\left(A-A_{w}\right)}-1\right)^{2}\left(\frac{v}{1-\beta}\right)^{2} .
$$

When air flows through the water film, the cross-sectional area at the time when the water film breaks is the minimum shrinkage cross section; that is, $\varepsilon=1$. Equation (14) can be simplified to

$$
\Delta P_{1}=\frac{1}{2} \rho\left(\frac{A}{\left(A-A_{w}\right)}-1\right)^{2}\left(\frac{v}{1-\beta}\right)^{2} .
$$

The effective area of the water film in the gap can be expressed by the equivalent wire diameter:

$$
A_{w}=\frac{\pi}{4} d^{2}(1-\beta) .
$$

Replacing equation (16) with equation (15) gives

$$
\Delta P_{1}=\frac{1}{2} \rho\left(\frac{A}{\left(A-(\pi / 4) d^{2}(1-\beta)\right)}-1\right)^{2}\left(\frac{v}{1-\beta}\right)^{2} .
$$

The theoretical calculation of dust removal assumes that the water mist is uniformly attached to the wire surface and flows owing to gravity. According to Figure 1, the equivalent diameter $d$ of the wire is the sum of the diameter $d_{s}$ of the wire and annular water mist component that is attached to the wire surface $[22,23]$.

Figure 1 shows that there is a relationship between $d$ and $d_{s}$ :

$$
\frac{\pi}{4}\left(d^{2}-d_{s}^{2}\right)=\frac{V_{s} \beta_{w}}{L_{s}},
$$

where $V_{s}$ is the plate volume $\left(\mathrm{m}^{3}\right)$ and $L_{s}$ is the total wire length $(\mathrm{m})$, the latter of which can be expressed as [24,25]

$$
L_{s}=\frac{4 \beta_{s} V_{s}}{\pi d_{s}^{2}} .
$$

Substituting equation (18) into equation (19) gives

$$
d=d_{s} \sqrt{1+\frac{\beta_{w}}{\beta_{s}}}=d_{s} \sqrt{\frac{\beta}{\beta_{s}}} .
$$

Combined with equations (17) and (20), the following can be used to calculate the inertia resistance at both ends of the wire with respect to fluid density, velocity, wire diameter, clearance area, and wet wire filling rate:

$$
\Delta P_{1}=\frac{1}{2} \rho\left(\frac{A}{\left(A-(\pi / 4)\left(d_{s}\left(\beta / \beta_{s}\right)\right)^{2}(1-\beta)\right)}-1\right)^{2}\left(\frac{\nu}{1-\beta}\right)^{2} .
$$

The total resistance in the dedusting process of a vibrating chord grid is as follows:

$$
\Delta P=\Delta P_{1}+f \frac{1}{2} \rho\left(\frac{A}{\left(A-(\pi / 4)\left(d_{s}\left(\beta / \beta_{s}\right)\right)^{2}(1-\beta)\right)}-1\right)^{2}\left(\frac{v}{1-\beta}\right)^{2}+\frac{4 \pi v\left(\left(7.13 \times 10^{-3} / T+110.4\right) \times(T / 288.15)^{(3 / 2)}\right)}{-(1 / 2)-(1 / 2) \ln \beta_{s}+\left(\beta^{2} / 1+\beta^{2}\right)} .
$$




\section{Results and Discussion}

3.1. Experimental System and Scheme. Dust-containing air in the return-air roadway of a mine is simulated through a vibrating chord grid dedusting system, as shown in Figure 3. The experimental system is composed of the following subsystems: dust, ventilation, spray, vibrating chord grid dust removal, and drainage. The vibrating string grid dust removal subsystem located in the middle of the apparatus is mainly composed of the vibrating string steel wire, water mist, and water film attached to the wire. The roadway model is made of $10 \mathrm{~mm}$ thick high-strength plexiglass to facilitate observation.

The dust subsystem of the experimental system is composed of an AG410 dry powder aerosol diffuser and 150-mesh mineral dust. The dedusting fan in the ventilation subsystem is GP-315, which provides stable air flow for the experiments. A TSI9565-P multifunction anemometer is used to measure air speed. The spray subsystem is composed of a SS-10w stainless steel nozzle, BPZ75/12 high-pressure pump, rotor flowmeter, DX801XB00150 digital pressure gauge, and a water supply pipe, which provide water and air pressure and water and gas flow under different working conditions. The vibrating grid plate in the vibrating chord grid dust removal subsystem has two specifications: one is made of steel wire with diameters of 0.35 and $0.75 \mathrm{~mm}$, and the other is made of steel wire with diameters of 0.35 and $0.1 \mathrm{~mm}$ and a filter area of $590 \times 550 \mathrm{~mm}$. The water mist and film attach to the vibrating wire grid plate. The drainage subsystem is set at the bottom of the roadway model. Droplets are ejected from the nozzle, flow from the vibrating grid plate, and are discharged from the drain at the model bottom. The wet chord grid filter dedusting section (where the spray dust removal system is located) is made of high-strength plexiglass and other sections are made of stainless steel.

\subsection{Experimental Scheme and Results}

3.2.1. Experimental Scheme. Tap water is used as the working medium. Considering the actual situation of mine water pressure system and limited water source, the equipment pressurizes the water to five pressure conditions commonly found in mines: $0.3,0.5,0.7,0.9$, and $1.1 \mathrm{MPa}$. Considering that the engineering application site is mine return-air roadway, according to the actual situation of the project site, combined with the needs of the experiment, the wind speed is set to $3,3.5,4,4.5$, and $5 \mathrm{~m} / \mathrm{s}$. Two kinds of vibrating wire grid dust removal resistance are studied using different filling rates, wind speeds, and water pressure conditions. The resistance measurement points are arranged prior to and after the experiments. Local and frictional resistance loss is considered negligible. To improve measurement accuracy, the data collection points were symmetrically arranged using the geometric center of the chord grid as the coordinate origin. Fifteen pairs of measuring points were arranged on either side of the grid for a total of 30 points. Owing to space limitations, this study only analyzes pairs of measuring points that are $3.05 \mathrm{~m}$ from the geometric center for a total of 50 data groups.

3.2.2. Analysis of Experimental Results. (1) Effect of wind speed on dust removal resistance: the grid resistance increases from $32.5 \%$ and $77.8 \%$ when the filling rate is increased from 3 to $5 \mathrm{~m} / \mathrm{s}$ under a spray pressure of $0.3 \mathrm{MPa}$. Figure 4(a) compares dedusting resistance at variable filling rate and constant spray pressure. Larger filling rates are associated with smaller dust removal resistance. This is because small filling rates more easily produce vortex phenomena owing to the inverse gradient pressure, which increases the inertia resistance. The dedusting resistance increases linearly with increasing wind speed. When the wind speed is $3 \mathrm{~m} / \mathrm{s}$, the resistance of $32.5 \%$ filling rate is $35.8 \mathrm{~Pa}$ and a $77.8 \%$ filling rate at the minimum resistance is $27.5 \mathrm{~Pa}$. The grid resistance under filling rates of $32.5 \%$ and $77.8 \%$ increases when the spray pressure increases from 0.3 to $0.5 \mathrm{MPa}$ under a wind speed of $5 \mathrm{~m} / \mathrm{s}$ (Figure $4(\mathrm{~b})$ ). Dust removal resistance is larger when using a filling rate of $32.5 \%$ compared with a $77.8 \%$ filling rate to a maximum of $6 \mathrm{~Pa}$. This behavior continues with increasing spray pressure; however, the extent of resistance change decreases (Figures 4(c)-4(e)). The maximum resistance increment is $8.8 \mathrm{~Pa}$ for a filling rate of $77.8 \%$ at a wind speed of $4 \mathrm{~m} / \mathrm{s}$ and $2.8 \mathrm{~Pa}$ at a wind speed of $4.5 \mathrm{~m} / \mathrm{s}$. The average value of vibrating chord grid increase is $3.78 \mathrm{~Pa}$ when the filling rate is $77.8 \%$.

The results show that resistance increases with spray wind speed at constant spray pressure. The dust removal resistance with a filling rate of $77.8 \%$ is substantially smaller than that with a filling rate of $32.5 \%$. The dedusting resistance is smallest when the wind speed is $3 \mathrm{~m} / \mathrm{s}$, which combines with the actual demand for wind speed during mine production. The optimal wind speed is $3.5 \mathrm{~m} / \mathrm{s}$, under which the dust removal cost is low and little influence is exerted on the mine ventilation system, making these conditions suitable for application in mine dust removal. The dedusting resistance and maneuverability should be taken into account to ensure dedusting efficiency. In summary, a wind speed of $3.5 \mathrm{~m} / \mathrm{s}$ and filling rate of $77.8 \%$ are recommended for application in engineering projects.

(2) Effect of spray pressure on dust removal resistance: resistance characteristics of the grid plate over a spray pressure range of 0.3 to $1.1 \mathrm{MPa}$ are shown in Figures 5(a)-5(e).

(1) At the position $(3.05,0)$, larger spray pressure yields higher resistance. Dedusting resistance increases continuously with increasing wind speed. At a wind speed of $3 \mathrm{~m} / \mathrm{s}$, the resistance is lower when the filling rate is $77.8 \%$ compared with $32.5 \%$ but the difference is only $8.3 \mathrm{~Pa}$. This difference increases to $23 \mathrm{~Pa}$ at a wind speed of $5 \mathrm{~m} / \mathrm{s}$.

(2) The grid under a fixed filling rate has higher resistance under high wind speeds than that at low wind speed. However, in practical applications, the wind 


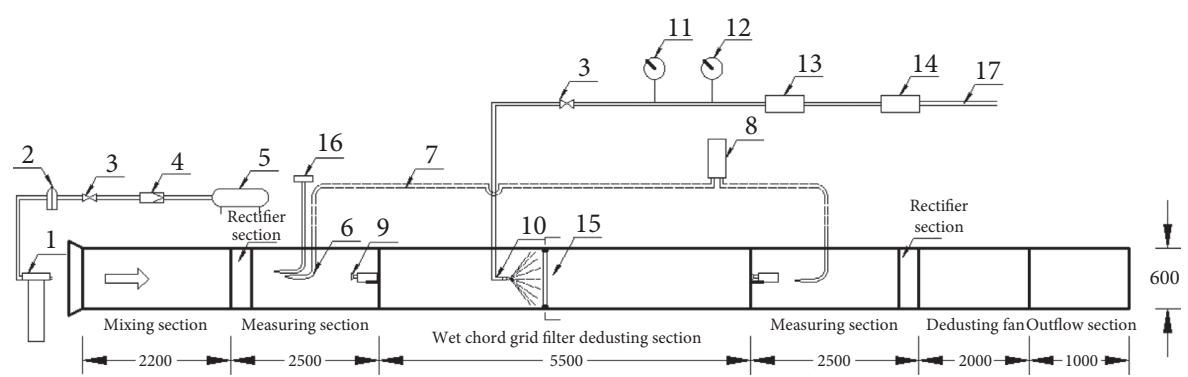

Figure 3: Test system of the wet vibrating string grille filter and dust removal. (1) AG410 dry powder aerosol diffuser; (2) rotor flowmeter; (3) gate valve; (4) pressure switch; (5) air compressor; (6) pitot tube; (7) rubber tube; (8) TSI pressure gauge; (9) CCZ-20 dust sampler; (10) SS10w stainless steel nozzle; (11) DX801XB00150 digital pressure gauge; (12) intelligent electromagnetic flowmeter. (13) BPZ75/12 highpressure pump; (14) water storage tank; (15) pure manual self-made vibrating grid plate; (16) TSI9565-P multifunction anemometer; (17) connection with the municipal pipe network.

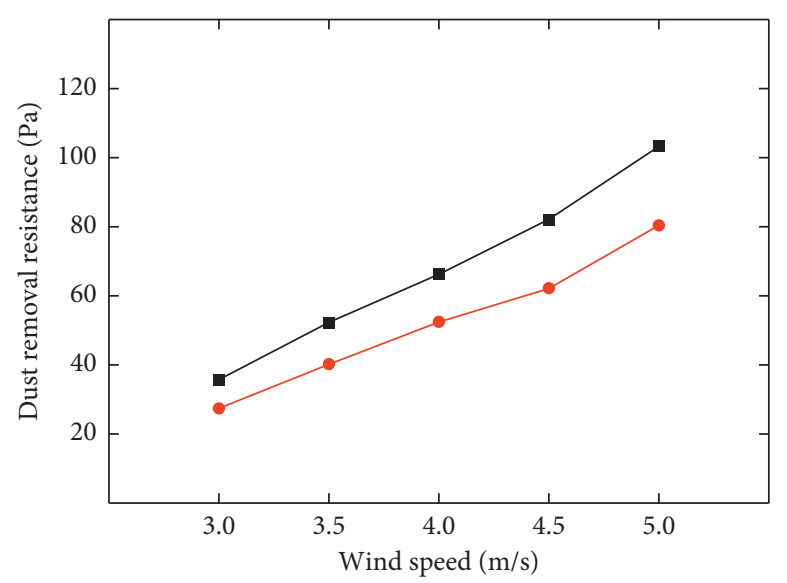

Filling rate $\beta=32.5 \%$ dust removal resistance - Filling rate $\beta=77.8 \%$ dust removal resistance

(a)

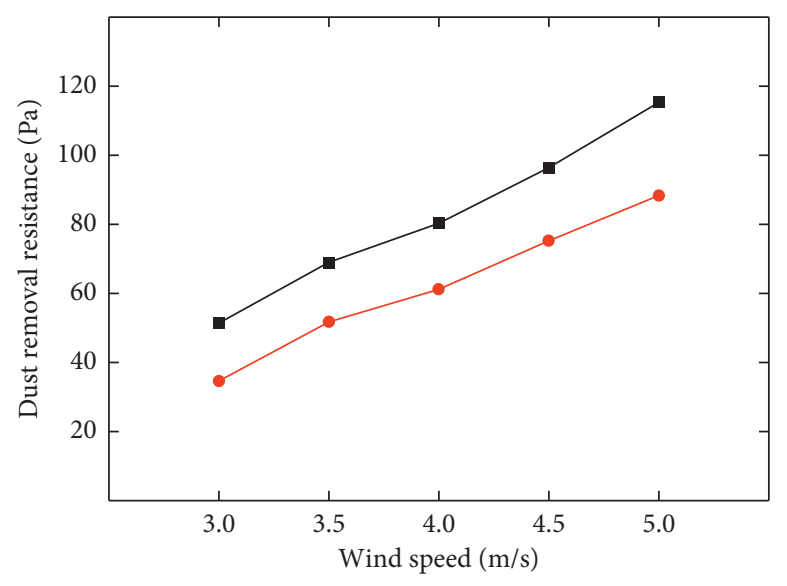

- Filling rate $\beta=32.5 \%$ dust removal resistance - Filling rate $\beta=77.8 \%$ dust removal resistance

(c)

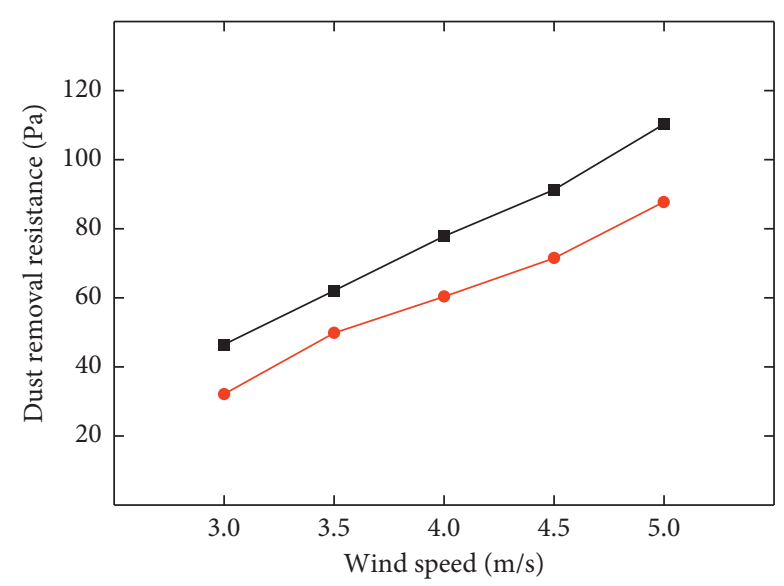

$\rightarrow$ Filling rate $\beta=32.5 \%$ dust removal resistance - Filling rate $\beta=77.8 \%$ dust removal resistance

(b)

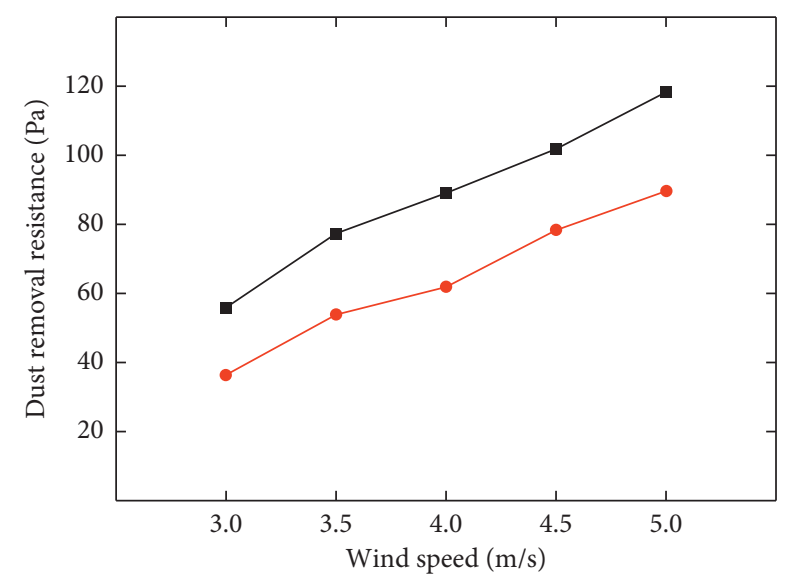

- Filling rate $\beta=32.5 \%$ dust removal resistance

- Filling rate $\beta=77.8 \%$ dust removal resistance

(d)

FIgUre 4: Continued. 


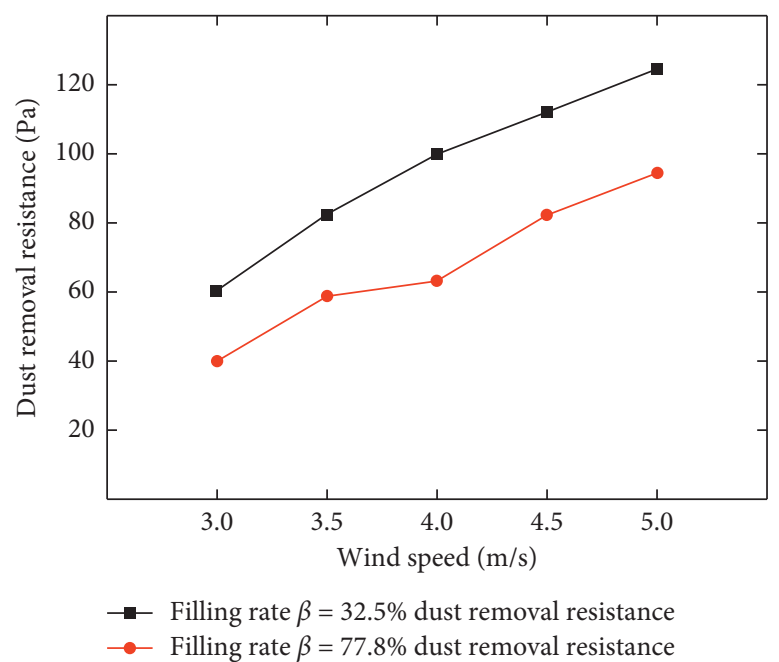

(e)

Figure 4: Spray pressure and variable wind speeds. (a) $0.3 \mathrm{MPa}$. (b) $0.5 \mathrm{MPa}$. (c) $0.7 \mathrm{MPa}$. (d) $0.9 \mathrm{MPa}$. (e) $1.1 \mathrm{MPa}$.

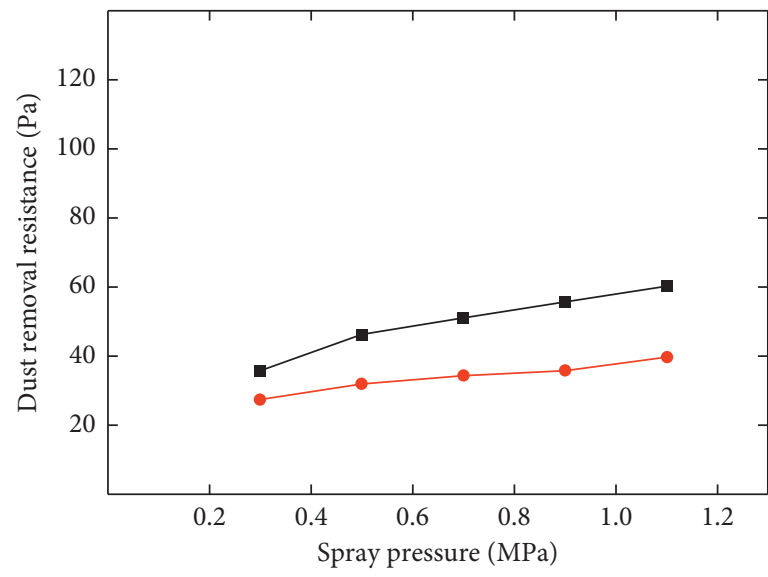

Filling rate $\beta=32.5 \%$ dust removal resistance Filling rate $\beta=77.8 \%$ dust removal resistance

(a)

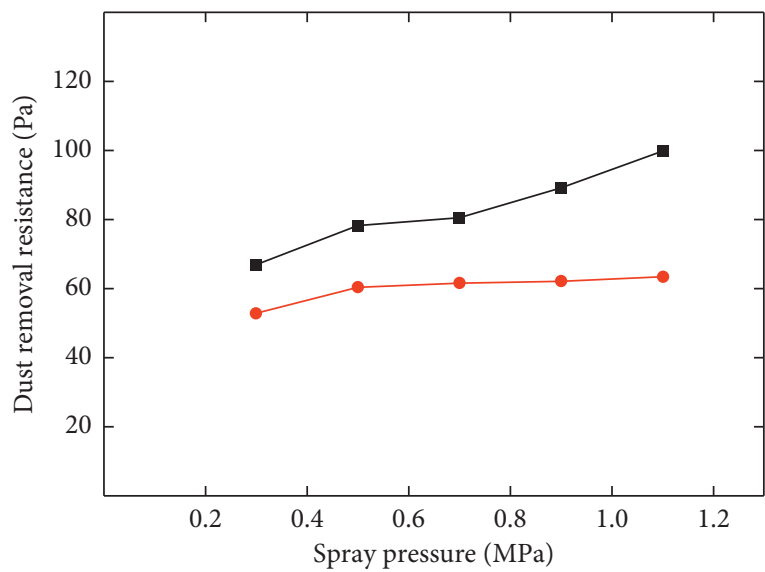

Filling rate $\beta=32.5 \%$ dust removal resistance Filling rate $\beta=77.8 \%$ dust removal resistance

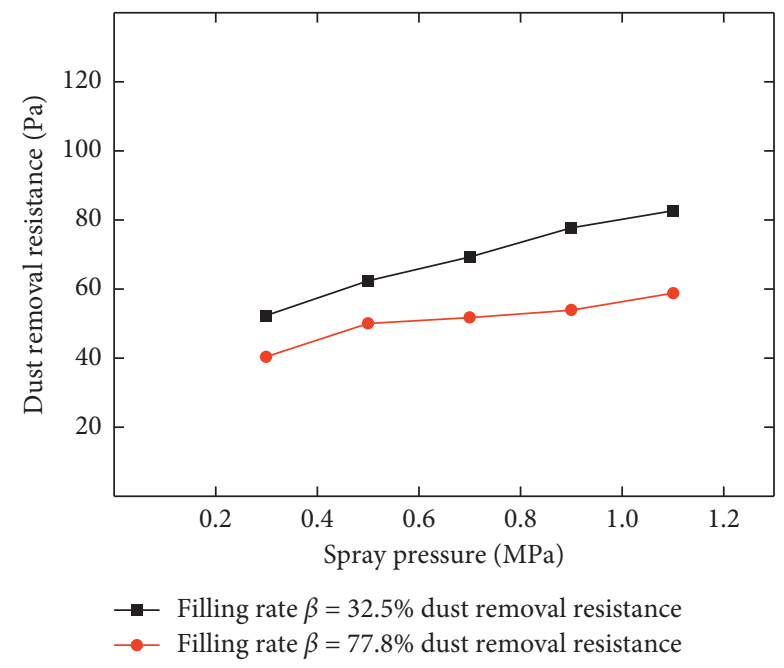

(b)

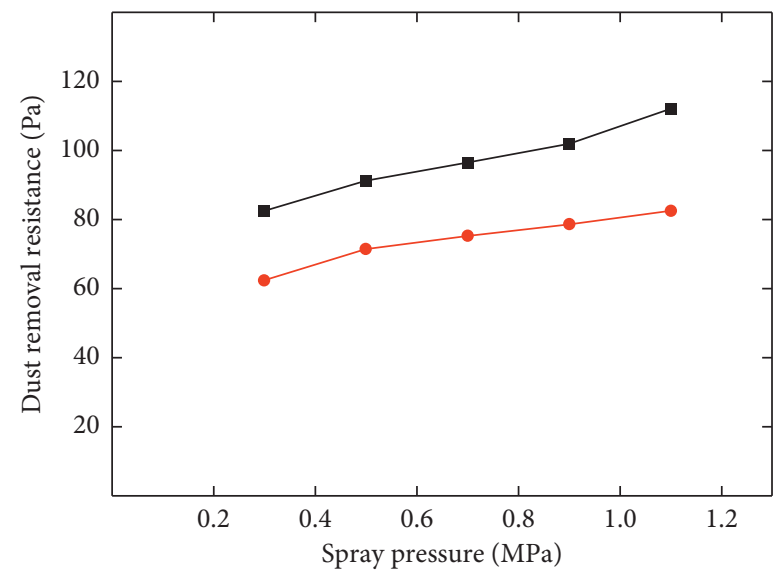

Filling rate $\beta=32.5 \%$ dust removal resistance Filling rate $\beta=77.8 \%$ dust removal resistance

(c)

(d)

Figure 5: Continued. 


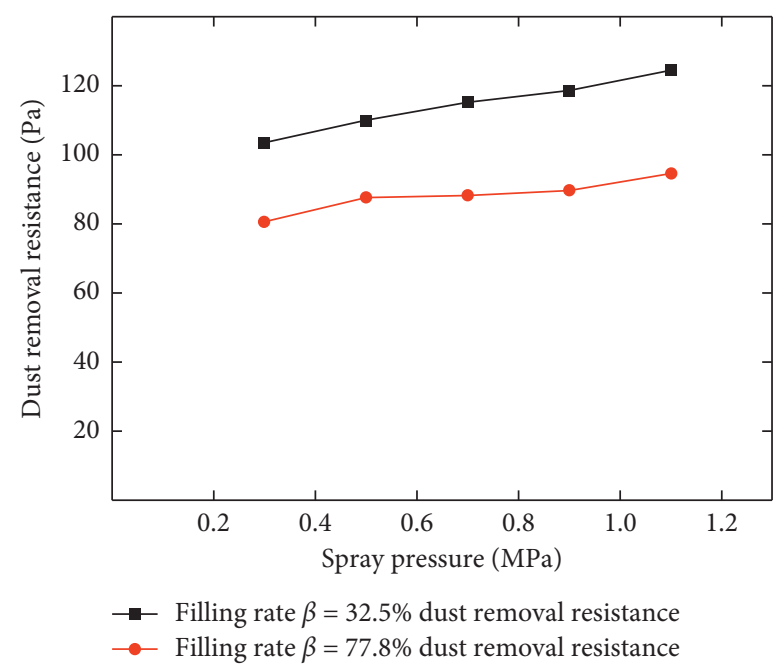

(e)

FIGURE 5: Wind speed and variable spray pressure.

(a) $3 \mathrm{~m} / \mathrm{s}$. (b) $3.5 \mathrm{~m} / \mathrm{s}$. (c) $4 \mathrm{~m} / \mathrm{s}$. (d) $4.5 \mathrm{~m} / \mathrm{s}$. (e) $5 \mathrm{~m} / \mathrm{s}$.

speed cannot be too low owing to practicality and to not disrupt normal production.

(3) For a grid plate with a filling rate of $77.8 \%$ and wind speed of $3 \mathrm{~m} / \mathrm{s}$, the average dust removal resistance is $34.02 \mathrm{~Pa}$. With the change of wind speed to $3.5 \mathrm{~m} / \mathrm{s}$, the average dust removal resistance is $50.82 \mathrm{~Pa}$. The average resistance is least affected when the spray pressure is $0.3 \mathrm{MPa}$, followed by $17.1 \mathrm{~Pa}$ at $0.7 \mathrm{MPa}$. Water film formation is not conducive under spray pressure conditions of $0.3 \mathrm{MPa}$. However, resistance substantially increases under other spray pressures with an optimum value of $0.7 \mathrm{MPa}$.

The results presented here show that dust removal resistance increases slightly with increasing spray pressure. A spray pressure of $0.3 \mathrm{MPa}$ yields the smallest resistance, which matches dust removal efficiency requirements. However, the dedusting cost is lower when the spray pressure is $0.7 \mathrm{MPa}$ and this setup has little influence on the mine ventilation system, which makes it suitable for use in mines. Considering the control of water consumption and atomization pressure, the dust removal resistance and feasibility should be taken into account based on long-lasting dedusting efficiency. A spray pressure of $0.7 \mathrm{MPa}$ and filling rate of $77.8 \%$ are recommended.

\subsection{Comparison between Calculations and Experimental} Data. To verify the accuracy of the theoretical expression of dust removal resistance, we analyze a system with a spray pressure of $0.7 \mathrm{MPa}$, wind speed between 3 and $5 \mathrm{~m} / \mathrm{s}$, and filling rate of $77.8 \%$. Compared with the experimental data (Figure 5(c)), the dust reduction remains constant at a spray pressure of $0.7 \mathrm{MPa}$ (Figure 6). The theoretical and experimental resistance values increase similarly with wind speed, which verifies that the theoretical curve can correctly describe resistance characteristics. However, differences between experimental and theoretical values become more

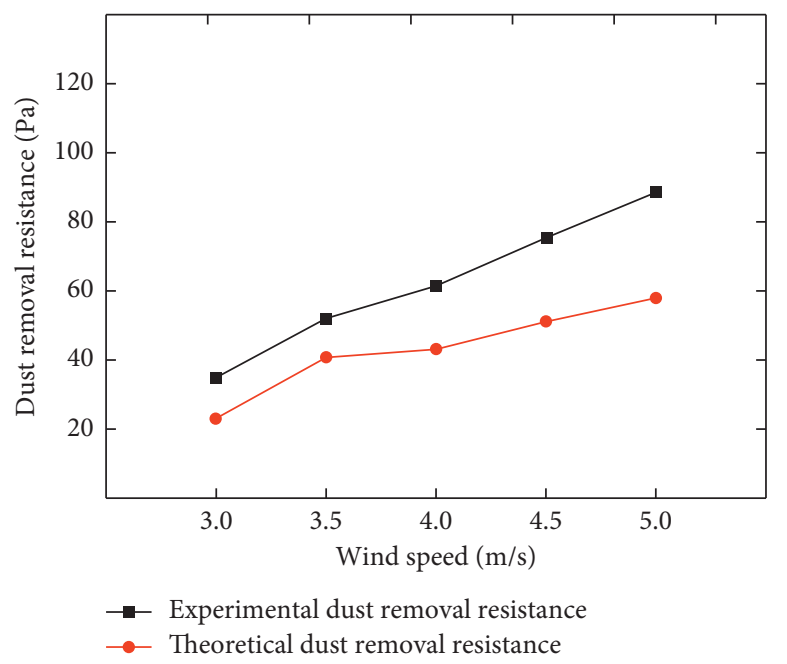

FIgURE 6: Comparison between theoretical resistance and experimental resistance under a water pressure of $0.7 \mathrm{MPa}$.

apparent under higher wind speeds. This is because the theoretical value assumes ideal conditions, where only the static chord grid parameters and air flow velocity are considered, whereas in the experiments, the vibration amplitude of the chord grid increases with wind speed and the water film is compressed, resulting in a larger resistance gap.

\section{Conclusion}

(1) It is assumed that there is no heat exchange on the surface of vibrating wire, and the water film of chord grid is uniformly distributed. By using the continuity equation and momentum equation, the friction resistance and inertia resistance are derived. The total dust removal resistance of the vibrating string grid is obtained when the dust flows around the wet vibrating wire. 
(2) On the experimental platform, under the working conditions of constant spray pressure and wind speed of 3-5 $\mathrm{m}$ for two different specifications of resonant chord grid, it is concluded that the dedusting resistance of vibrating chord grid with high filling rate is less than that of low filling rate and that the dedusting resistance of vibrating string grid with high filling rate is less than that of low filling rate under the condition of constant wind speed and spray pressure of $0.3-1.1 \mathrm{MPa}$. The change of the experimental test value is about the same as that calculated by the theoretical formula.

(3) According to the theoretical derivation and experimental data, a chord grid with a filling rate of $77.8 \%$ should be adopted in the mine return-air roadway with a spray pressure of $0.7 \mathrm{MPa}$ and optimum air speed of $3.5 \mathrm{~m} / \mathrm{s}$.

\section{Data Availability}

The raw data are available from the corresponding author upon request.

\section{Conflicts of Interest}

The authors declare that they have no conflicts of interest.

\section{Acknowledgments}

The authors thank Esther Posner, Ph.D., from Liwen Bianji, Edanz Editing China (http://www.liwenbianji.cn/ac), for editing the English text of a draft of this manuscript. This paper was supported by the Excellent Youth Project of Hunan Provincial Department of Education (no. 19B223), the National Natural Science Foundation of China (no. 51774134), the Hunan Provincial Natural Science Foundation of China (no. 2019JJ60044), and the 2017 Hunan Provincial Graduate Research Innovation Project of China (no. CX2017B649).

\section{References}

[1] Z. A. Jiang, J. H. Chen, and M. Wang, "Numerical simulation on influencing factors of dust concentration in ore unloading station," Journal of China Coal Society, vol. 43, no. S1, pp. 185-191, 2018.

[2] Ministry of Environmental Protection of the People's Republic of China, General Administration of Quality Supervision, Inspection and Quarantine. GB3095-2012 Code for Environmental Air Quality, China Environmental Science Press, Beijing, China, 2012.

[3] L. Z. Jin, J. P. Li, and Y. F. Su, Theory of Mine Dust Prevention and Control, Science Press, Beijing, China, 2010.

[4] B. Xie, S. Li, H. Jin, S. Hu, F. Wang, and F. Zhou, "Analysis of the performance of a novel dust collector combining cyclone separator and cartridge filter," Powder Technology, vol. 339, pp. 695-701, 2018.

[5] S. Y. Hu, Q. Liao, and H. T. Wang, "Gas-solid two-phase flow at high-gassy fully mechanized within high gassy coal seam," Journal of China Coal Society, vol. 44, no. 12, pp. 3921-3930, 2019.
[6] F. B. Zhou, J. L. Li, S. H. Li et al., "Experimental investigation and application of dry-type filtering dust collection technology in fully mechanized excavation face," Journal of China Coal Society, vol. 42, no. 3, pp. 639-645, 2017.

[7] J. Krawczyk, "Change in dust collection efficiency of liquid collectors in conditions of dedusting liquid recirculation," Polish Journal of Chemical Technology, vol. 19, no. 4, pp. 1-7, 2017.

[8] W. R. Reed, S. Klima, M. Shahan et al., "A field study of a roof bolter canopy air curtain (2nd generation) for respirable coal mine dust control," International Journal of Mining Science and Technology, vol. 29, no. 5, pp. 711-720, 2019.

[9] G. Zhou, W. Nie, W. M. Cheng et al., "Influence regulations analysis of high-pressure atomization dust-settling to dust particle's microscopic parameters in fully mechanized caving coal face," Journal of China Coal Society, vol. 39, no. 10, pp. 2053-2059, 2014.

[10] V. Igor, R. Vladyslav, and A. Iasynetskyi, "Flue gas dedusting in venturi scrubbers at thermal power plants," Environmental Engineering\&Management Journal (EEMJ), vol. 17, no. 11, pp. 2515-2525, 2018.

[11] L. H. Gong, Y. M. Wang, and K. Liu, "Experimental study on the dust collection mechanisms of wet fiber grid," Journal of Liaoning Technical University (Natural Science Edition), vol. 2, no. 1, pp. 26-28, 2001.

[12] S. Y. Ge, The Research and Application of the High Pressure Atomizing and Wet Vibration Fiber Grid Dust Precipitation Technology, University of Science and Technology Beijing, Beijing, China, 2007.

[13] J. Zhao, Research and Application of Air Shaft Wet Resonance Grille Dust Removal Technology, Hunan University of Science and Technology, Xiangtan, China, 2015.

[14] G. Z. Zhang, Study on Experiment and Mechanism of Light Moving Wet Vibrating String Precipitator Ganzhou, Jiangxi University of Science and Technology, Ganzhou, China, 2012.

[15] X. S. Chen and Y. L. Zhang, "Analogy research on wet fiber layer filtration resistance of mine dust China," Environmental Science, vol. 34, no. 11, pp. 2721-2725, 2014.

[16] X. D. Xiang, Foundation of Aerosol Science and Technology, China Environmental Science Press, Beijing, China, 2012.

[17] X. S. Chen and H. Y. Gu, "Study on dust filtration efficiency about wet fiber layer progress in environmental science and technology," in Proceedings of the 2009 International Symposium on Environmental Science and Technology, pp. 841-847, Shanghai, China, June 2009.

[18] X. D. Xiang, Theoretical Technology and Application of Soot Fiber Filtration, Metallurgical Industry Press, Beijing, China, 2007.

[19] Z. N. Wu, Aerodynamics, Beijing University of Aeronautics and Astronautics, Beijing, China, 2016.

[20] L. H. Gong, Theoretical and Experimental Study on Dust Removal Technology of Wet Vibrating Fiber Grid, Northeast University, Shenyang, China, 2002.

[21] H. Q. Song, Engineering Fluid Dynamics and Environmental Application, Metallurgical Industry Press, Beijing, China, 2016.

[22] D. Prostański, "Use of air-and-water spraying systems for improving dust control in mines," Journal of Sustainable Mining, vol. 12, no. 2, pp. 29-34, 2013.

[23] M. Versteeg, An Introduction to Computational Fluid Dynamics: The Finite Volume Method, World Publishing Corporation, Beijing, China, 2nd edition, 2010.

[24] R. Rahimi and D. Abbaspour, "Determination of pressure drop in wire mesh mist eliminator by CFD," Chemical 
Engineering and Processing: Process Intensification, vol. 47, no. 9-10, pp. 1504-1508, 2008.

[25] B. Fabiano, F. Currò, A. P. Reverberi, and E. Palazzi, "Coal dust emissions: from environmental control to risk minimization by underground transport. an applicative casestudy," Process Safety and Environmental Protection, vol. 92, no. 2, pp. 150-159, 2014. 